\title{
Imagined stimuli: Imaginary effects?
}

\author{
JOHN PREDEBON and PETER WENDEROTH \\ University of Sydney, Sydney, Australia
}

Wallace (1984) reported the results of three experiments that apparently indicated a functional equivalence between imagery-induced and physically induced visual illusions. We argue that his findings can be plausibly explained in terms of demand characteristics present in the experimental situation.

Recently, Wallace (1984) reported three experiments that purported to demonstrate imagery-induced visual illusions. In particular, Wallace claimed to have shown that there is a relationship between the ability of observers to create vivid mental images and the magnitude of the imagery-induced illusions that they experience. It is our contention that demand characteristics (Orne, 1962) provide the most probable explanation of Wallace's data, despite his claims to the contrary.

In two of three experiments, Wallace (1984) investigated the Ponzo illusion (see Figure 1a), in which the inducing angle causes the flanked line nearer the vertex to appear to be longer than the physically equal lower line. Although the Wundt and Hering illusions were used in Experiment 3, it is sufficient for our purposes to restrict our comments to the Ponzo studies.

In each experiment, there were essentially two stimulus conditions. In one condition, subjects, using a millimeter rule as a guide, made magnitude estimates of the illusion in the complete display (Figure 1a). In a second condition, an independent group of observers made estimates of the illusion either while imagining the test line(s) - one or both-on to a real inducing angle (Experiment 1) or while imagining the inducing angle on to a real pair of test lines (Experiment 2). Subjects were selected from a student population on the basis of their responses to the Vividness of Visual Imagery Questionnaire, or VVIQ (Marks, 1973). For each of the two stimulus conditions, half of the subjects were classified as high imagers, and half were classified as low imagers.

In all experiments, all groups experienced similar illusions in the nonimagery conditions. However, in striking contrast, whereas all high imagers reported imaginary illusions that were comparable in magnitude to their "real" illusions, almost none of the low imagers were able to create the required image and, for those who could, illusions tended to be smaller than their "real" illusions.

How might one interpret these findings? Can it be concluded, with Wallace (1984), that, in the case of high imagers, "for all intents and purposes, an imagined event

The authors' mailing address is: Department of Psychology, University of Sydney, N.S.W. 2006, Australia.
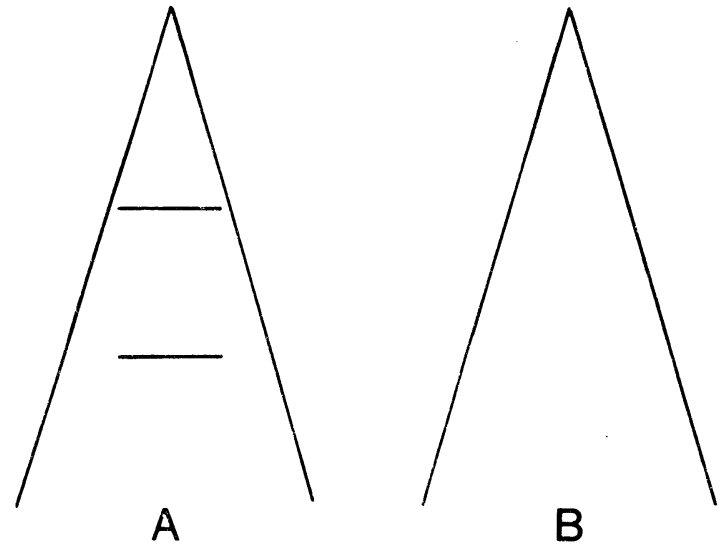

Figure 1. Ponzo illusion (a) and empty angle stimulus (b).

was equivalent to a physically present stimulus" and that, "for low imagers, this was not the case" (p. 162)? Or, can the data better be understood in terms of demand characteristics, including experimenter bias and tacit knowledge? In numerous other cases, demand characteristics have been invoked to explain the results of studies that have allegedly shown a functional equivalence between effects induced physically and those induced via imagination (e.g., Harris, 1982; Intons-Peterson, 1983; Intons-Peterson \& White, 1981; Mitchell \& Richman, 1980). Wallace (p. 158) rejected this interpretation of his data on the grounds that, if demand characteristics were the explanation, then "many more low imagers would have reported the presence of an imagined stimulus and the subsequent appearance of an illusion." Also, Wallace ensured that the experimenter was blind to the subjects' VVIQ scores and the experimental hypothesis, that subjects were unaware of the illusion direction, and that all attempts were made to minimize the role of demand characteristics "by the manner in which the subjects were asked to respond to stimuli, imagined or real"' (p. 161).

In a different context, Harris (1982) noted that scores on the VVIQ and the Stanford Hypnotic Susceptibility Scale are positively correlated (Hoen, 1978). To the extent that susceptibility to hypnosis and susceptibility to demand characteristics are correlated also, it seems necessary to explore a little further the possibility that high im- 
agers' susceptibility to demand characteristics might account for Wallace's (1984) results.

In our view, demand characteristics must provide the explanation of Wallace's (1984) Experiment 1, in which subjects were shown the empty angle stimulus (Figure 1b) and "were told to imagine two horizontal lines of equal length, one above the other. The experimenter indicated to the subjects where their two lines were to be placed via imagery" (p. 157). If the subject reported being able to image the lines, the question then asked was whether the lines appeared equal in length and, if not, which appeared longer and by how much.

This procedure, we contend, involves a novel variation of the El Greco fallacy (Howard, 1982). It was once held that El Greco drew long, thin figures because of his astigmatism. This is a fallacy, because if El Greco saw a normal person as long and thin, he would reproduce that person to appear long and thin by drawing a normal person. Similarly if, as Wallace (1984) argued, imaged lines are subject to illusions, then when a subject imaged two equal-appearing lines on the inducing angle, the lines would already be subject to the inducing effect of the angle and therefore could not possibly appear to be unequal. A better procedure might have been to have the subjects image two equal lines (pretest) and then to introduce the inducing element to quantify its effect, or to remove the inducing element after imagery to measure any change. Wallace's procedure, however, implies that subjects simultaneously saw the two imaged lines as equal and unequal, a finding that carries startling implications concerning the non-Euclidean nature of imaged space.

An alternative, and perhaps more plausible, interpretation is to regard Wallace's (1984) Experiment 1 as a classical example of a control or "catch" condition for the evaluation of the role of demand characteristics in such experiments. That is, if the subject followed instructions and did image two equal-appearing lines, then the sole factor that could lead to reports of inequality is the suggestion, implicit or otherwise, of inequality.

We contend, on the basis of other research (IntonsPeterson \& White, 1981) that it is likely that the experimenter unwittingly cued subjects to respond in the appropriate manner. To be sure, experimenters were "blind" to the particular hypothesis under test, but they were aware of the Ponzo illusion and inadvertently could have signaled expected responses to subjects. This, together with the assumption that high imagers are more susceptible than low imagers to demand characteristics, seems to us to provide a plausible demand-characteristics account of Wallace's (1984) findings.

In addition, it should be noted that no attempt was made to include a control group who merely were told about the stimulus configuration and were asked what sort of illusion might be expected: It seems not unreasonable to suggest that the expected direction of the simple Ponzo illusion is obvious. A much more convincing demonstration would involve nonobvious results, such as the reversal of various illusions with slight changes in stimulus parameters, or the angular function, say, of the tilt illusion. Would the imaged tilt illusion peak when the inducing and test lines differ in orientation by $10^{\circ}-15^{\circ}$, as does the physical effect?

In conclusion, we do not seek to deny the possibility of inducing visual illusions via imagery. It may well be the case that imaged contours, like cognitive or subjective contours, behave like real lines. We do contend, however, that Wallace (1984) failed to provide an unambiguous test of the purported equivalence between physically induced and imagery-induced illusions.

\section{REFERENCES}

HARRIS, J. P. (1982). The VVIQ and imagery-induced McCollough effects: An alternative analysis. Perception \& Psychophysics, 32, 290-292.

HOEN, P. T. (1978). Effects of hypnotizability and visualizing ability on imagery-mediated learning. International Journal of Clinical and Experimental Hypnosis, 26, 45-54.

HowARD, I. P. (1982). Human visual orientation. New York: Wiley. InTONS-PETERSON, M. J. (1983). Imagery paradigms: How vulnerable are they to experimenters' expectations? Journal of Experimental Psychology: Human Perception and Performance, 9, 394-412.

Intons-Peterson, M. J., \& White, A. R. (1981). Experimenter naivete and imaginal judgments. Journal of Experimental Psychology: Human Perception and Performance, 7, 833-843.

MARKS, D. F. (1973). Visual imagery differences in the recall of pictures. British Journal of Psychology, 64, 17-24.

Mitchell, D. B., \& Richman, C. L. (1980). Confirmed reservations: Mental travel. Journal of Experimental Psychology: Human Perception and Performance, 6, 58-66.

ORNE, M. T. (1962). On the social psychology of the psychology experiment: With special reference to demand characteristics and their implications. American Psychologist, 17, 776-783.

WALLACE, B. J. (1984). Apparent equivalence between perception and imagery in the production of various visual illusions. Memory \& Cognition, 12, 156-162.

(Manuscript received February 12, 1985.) 